\title{
Comparative analysis of mitochondrial genome between UG93A and UG93B reveal common feature of 5'- end heterogeneity in mitochondrial genes of kenaf
}

\author{
Xiaofang Liao, ${ }^{1,2}$, Yanhong Zhao ${ }^{2}$, Aziz Khan ${ }^{1}$, Xiangjun Kong ${ }^{1}$, Bujin Zhou ${ }^{1}$, Min Li ${ }^{1}$, \\ Meiling Wei ${ }^{1}$, Shuangshuang Peng ${ }^{1}$, Fazal Munsif ${ }^{1}$, Ruiyang Zhou ${ }^{{ }^{*}}$ \\ ${ }^{1}$ Key Laboratory of Plant Genetic and Breeding, College of Agriculture, Guangxi University, Nanning \\ 530005, China \\ ${ }^{2}$ Cash Crop Institute of Guangxi Academy of Agricultural Sciences, Nanning, 530007, China \\ *Correspondent: ruiyangzhou@ aliyun.com;
}

\begin{abstract}
Plant cytoplasmic male sterility (CMS) being maternal phenomenon trait that result from pollen abortion and closely linked with mitochondrial DNA rearrangement in many crops including kenaf. However, the molecular mechanism in kenaf is poorly known. In present work, we described the mitochondrial genome in isonuclear CMS line UG93A and its maintainer line UG93B. Findings of the current study revealed that a total of 398 SNPs and $230 \mathrm{InDels}$ were identified in UG93A mtDNA. Total of 26 SNPs variations and three InDels were identified in the coding region of atp6, indicating its active role in mitochondrial genome re-arrangement. Northern blot analysis showed that the transcripts of atp1, atp4, atp6, cox3 and sdh4 in $\mathrm{F}_{1}$ were consistent with UG93A but different for UG93B. The transcript of atp9 was found similar between UG93B and $\mathrm{F}_{1}$ while different for UG93A, which depict that atp 9 may be regulated by nuclear genes in $\mathrm{F}_{1}$ hybrid. The expression of atp 9 in UG93A was substantially lower compared with UG93B, suggesting its key role for energy supplying in microspore development of kenaf. Circularized RNA (CR)-RT-PCR revealed that mitochondrial RNAs with heterogeneous 5'- ends but uniform 3' - ends are common feature in kenaf mitochondrial genes, and the promoter architecture analysis showed that the promoter sequences in kenaf mitochondrial genome are highly diverged in comparison to those in other plants. Our data highlight that the translation of mitochondrial genes in kenaf is closely associated with heterogeneity of the 5'- end of plant mRNA. The present result provides the basic information in understanding the transcription of kenaf mitochondrial genome and can be used as reference in other plants.
\end{abstract}

Keywords: kenaf (Hibiscus cannabinus), cytoplasmic male sterility, mtDNA resequencing, 
transcription, circularized RNA (CR)-RT-PCR, 5'-heterogeneity

\section{Introduction}

Angiosperm mitochondrial (mt) genomes are complex and differ substantially in size ranging from $208 \mathrm{~kb}$ in Brassica hirta (Kubo et al., 2000) to 11.3 Mb in Silene conica (Sloan et al., 2012). The mt genomes of angiosperms usually not only reveal phylogenetic relationships among plant species but also intraspecific differentiation in cytoplasm (Fujii et al., 2010). The Cucurbitaceae genomes ranges from $380 \mathrm{~kb}$ (Citrullus lanatus) to 2,400 kb (Cucumis melo) due to the amount of chloroplast sequence $(113 \mathrm{~kb})$ transfers and short sequences duplications $(370 \mathrm{~kb})$ in larger Cucurbita mitochondrial genome (Alverson et al., 2010; Rodriguez-Moreno et al., 2011). In CMS mitochondrial genomes, LD-CMS, derived from Oryza sativa, has a size of $434 \mathrm{~kb}$ while that of CW-CMS has $559 \mathrm{~kb}$ derived from Oryza rufipogon due to genomic dynamic rearrangements (Fujii et al., 2010). Despite substantial variation in size and physical mapping properties, plant mitochondria exhibited significant conservation in angiosperm genes, including 37-83 protein coding, tRNA and rRNA genes (Adams et al., 2002). The shuffling of mitochondrial DNA(mtDNA) sequences by recombination, repeat sequences and noncoding sequences has vital in $\mathrm{mt}$ genome evolution by altering gene organization and creating chimeric genes (Hanson and Bentolila, 2004; Chen and Liu, 2014).

Cytoplasmic male sterility (CMS) is a common phenomenon causing incompatibility between nucleus and mitochondria and has been found in various plant species (Liu et al., 2011; Luo et al., 2013; Okazaki et al., 2013; Heng et al., 2014; Reddemann and Horn, 2018). CMS systems are not only useful genetic tool for hybrid crop breeding, but also ideal models for studying the genetic interaction and cooperative function of mitochondrial and nuclear genomes in plants (Hanson and Bentolila, 2004). Plant CMS may be caused by mutations, rearrangements or recombinations in the mitochondrial genome and novel chimeric genes deduced from mtDNA rearrangements have been identified in several plants (Hanson and Bentolila, 2004). In rice WA-CMS, a new mitochondrial chimeric protein WA352, interacts with the nuclear-encoded mitochondrial protein COX11, inhibiting its function in peroxide metabolism and triggering premature tapetal programmed cell death, leading to pollen abortion (Luo et al., 2013). A CMS protein, ORFH79, binding to complex III, decreases its enzymatic activity in mitochondria and increases the reactive oxygen species (ROS) content through interactions with P61, resulting in pollen abortion in rice HL-CMS (Wang et al., 2013). A toxic protein $O R F 288$, co-transcribe with atp6 and make transgenic plant fail to 
anther development, indicating its key role in Brassica juncea male sterility (Jing et al., 2012, Heng et al., 2018).

Mitochondria, chloroplast and other organelles contain its own genomes, however, their gene expression systems are distinct from other eukaryotes. Recently, studies have documented that the significance of regulating mitochondrial genes expression at RNA level, including regulation of multiple transcription initiation site at 5'-end of plant mitochondrial genes (Kuhn et al., 2002; Kuhn et al., 2005), formation of the 5' - and 3' - ends of mitochondrial mRNA, stabilization of stem-loop structures and degradation of polyadenylation of mitochondrial mRNA (Kuhn et al., 2001; Forner et al., 2007). Several nuclear-encoded proteins regulated plant mitochondrial genes expression. The pentatricopeptiderepeat (PPR) protein of PPR336 gene has been shown to closely related with polysomes in mitochondria, which could be involved in translation of plant mitochondrial genes (Uyttewaal et al., 2008). Another PPR protein of MPPR6 gene that interacts with 5'UTR of rps 3 mRNA is involved in 5' maturation and translational initiation of rps3 mRNA in maize. Functional deficiency of MPPR6 results in a considerable reduction of rps 3 translation (Manavski et al., 2012).

Kenaf (Hibiscus cannabinus) is an important fiber crop that is widely used in paper-making and weaving (Monti and Alexopoulou, 2013). Hybrid kenaf has attracted great interest due to its higher yield with better fiber quality and stress resistance (Tao et al., 2008; Zhou et al., 2008). The first cytoplasmic male sterile line in kenaf was bred from wild UG93, which then used as common way to produce $F_{1}$ hybrid seeds (Zhou, 2002). CMS is an important factor in plant heterosis utilization and its mechanism of cytoplasmic male sterility is closely related to $\mathrm{mt}$ genome (Hanson and Bentolila, 2004). Sequencing of kenaf mt genome would not only be helpful for studying the evolutionary processes but could also clarify the inheritance mechanism for CMS in kenaf and the first mitochondrial genome of kenaf has been sequenced by Liao et al. (2018). Despite increasing interest in kenaf CMS, not much is known about its molecular mechanisms (Zhao et al., 2013; Zhao et al., 2015; Liao et al., 2016; Zhao et al., 2016). Efforts should be made to elucidate the factors underlying the mechanism of this important trait for hybrid breeding in kenaf. In present study, sequencing of UG93A mt genome in kenaf was determined and Northern blot analyses were used to compare differential transcription among UG93A, UG93B and $F_{1}$ (UG93A/UG93R). Circularized RNA (CR)-RT-PCR was used to analyze the multiple transcription initiation sites of mitochondrial genes. Our data provides a better understanding of evolutionary processes of 
kenaf $\mathrm{mt}$ genome. Furthermore, this study could offer useful information in understanding the transcription of kenaf mitochondrial genome and can be used as reference in other plants.

\section{Materials and methods}

\section{Plant materials}

Kenaf CMS line UG93A is a naturally mutant of UG93 variety. It was provided by Institute of Bast Fiber Crops, Chinese Academy of Agricultural Sciences. The maintainer line UG93B was bred from wild-type variety UG93, and $F_{1}$ (UG93A/UG93R) and was descendant of restorer UG93R hybridized with UG93A. These lines were belong to same nuclear background (UG93 nucleus) and hence considered the idealist materials for nuclear-cytoplasmic interaction investigations. This can assist in elimination of redundant genetic information from different nuclear background.

\section{Mitochondrial DNA isolation and Illumina Hiseq 2000 resequencing}

Mitochondria were isolated from kenaf CMS line UG93A and purified from 7-day-old etiolated seedlings using differential centrifugation and discontinuous (18\%, 23\% and 40\%) Percoll density gradient centrifugation according to Wilson and Chourey method (1984). Mitochondrial DNA isolation was performed according to Porebski et al. (1997) with modifications. Purified mitochondria were lysed with cetyltrimethylammonium bromide (CTAB) supplemented with $2 \%$ polyvinylpyrrolidone and $0.7 \% \beta$-mercaptoethanol (Solarbio, Beijing, China ) at $65{ }^{\circ} \mathrm{C}$ for $30 \mathrm{~min}$. The lysis solution was extracted 2 to 3 times with chloroform/isoamyl alcohol (24:1), and absolute ethyl alcohol was used to precipitate mtDNA. DNase-free water $(50 \mu \mathrm{L})$ was added to resuspend DNA pellets. The integrity, quality and concentration of UG93A mtDNA were analyzed using agarose gel electrophoresis, a NanoDrop 2000 (Thermo Scientific, Massachusetts, USA) and a Qubit fluorometer (Thermo Scientific, Massachusetts, USA), respectively. Extracted mtDNA of UG93A was prepared for next-generation sequencing (NGS) as follows: $5 \mu \mathrm{g}$ of UG93A mtDNA was randomly sheared into fragments using a Covaris S220 (Thermo Scientific, Massachusetts, USA) with an insertion size of approximately 200-500 base pairs (bp) to construct a paired-end sequencing library. Fragmented DNA was combined with End Repair Mix (Qiagen, Duesseldorf, Germany), followed by the addition of $\mathrm{A}$ at the 3'- end, and Illumina paired-end adaptor oligonucleotides were ligated to the sticky ends. The ligation mixture was purified with a QIAquick PCR Purification Kit (Qiagen, Duesseldorf, Germany). Several rounds of PCR 
amplification with the PCR Primer Cocktail and PCR Master Mix (Qiagen, Duesseldorf, Germany) were performed to enrich the adapter-ligated DNA fragments. DNA Clusters of PCR colonies were resequenced on a HiSeq 2000 sequencing platform using the recommended protocols from the manufacturer.

\section{Genome annotations and analyses}

Low-quality sequences in raw data were filtered with the FASTX toolkit (http://hannonlab.cshl.edu/fastx_toolkit), and clean reads were obtained according to Cox et al (2010). BLASTn analysis was performed to exclude contamination of the chloroplast and nuclear genome sequences. Sequences were assembled into contigs using Velvet and Oases software (Cox et al., 2010). Clean reads were mapped and aligned onto the reference mtDNA sequence of UG93B with BWA software (Li and Durbin, 2009). Genes encoding mitochondrial proteins and rRNAs were annotated using BLASTn and MITOFY (Alverson et $a l ., 2010)$ based on the known annotation of kenaf mitochondrial genes of UG93B. The tRNA genes were identified using tRNA scan-SE software (http://lowelab.ucsc.edu/tRNAscan-SE/).

\section{RNA Extraction and Northern Blot analysis}

Total RNAs were isolated from anthers at binucleate stage using a Quick RNA Isolation Kit (Hua yue yang, Beijing, China) according to the protocol supplied by the manufacturer. For RNA blotting analysis, approximately $30 \mu \mathrm{g}$ of total RNA was denatured and separated on a $1 \%$ denaturing formaldehyde agarose gel and transferred to a Hybond- $\mathrm{N}^{+}$nylon membranes (GE Healthcare Life Sciences, London, UK). PCR products were labeled with DIG-High Prime DNA Labeling and Detection Starter Kit II (Roche, Mannheim, Germany), and the operating procedures according to the protocol supplied by the manufacturer of DIG-High Prime DNA Labeling and Detection Starter Kit II (Roche, Mannheim, Germany). The prober primes are shown in supplementary (Table S3).

\section{Synthesis of cDNA and $q R T-P C R$ analysis}

Two developmental stages of buds (at uninucleate stage and binucleate stage) were chosen to analyze the expression of atp1, atp4, atp6, atp9 and cox3. Total RNA was isolated from the anthers of UG93A, UG93B, and $\mathrm{F}_{1}$ (UG93A/UG93R) with CTAB as described by Liu and He (2006). One microgram of total RNA was used in the reverse transcription reaction using the TransScript One-Step gDNA Removal and cDNA Synthesis SuperMix (TransGene Biotech, 
Beijing) to obtain $20 \mu \mathrm{L}$ of the resulting cDNA solution. The gene-specific primers for qRT-PCR were designed by Primer Premier 5.0 software. Quantification was performed with SYBR $^{\circledR}$ Premix Ex Taq ${ }^{\mathrm{TM}}$ (Tli RNaseH Plus) (Takara, Beijing, China) using a Bio-Rad CFX96 instrument (Bio-Rad, California, USA). The housekeeping gene Histone H3 (Histone3) of kenaf served as the internal reference. PCR cycling was denatured using a program of $95^{\circ} \mathrm{C}$ for $10 \mathrm{~s}$ and 40 cycles of $95^{\circ} \mathrm{C}$ for $5 \mathrm{~s}$ and $60^{\circ} \mathrm{C}$ for $30 \mathrm{~s}$. For each sample three biological and technical replicates were used. The relative expression was calculated according to formula $2^{-\triangle \Delta}{ }^{\mathrm{Ct}}$ for UG93A, UG93B, and $\mathrm{F}_{1}$ (UG93A/UG93R), with maintainer line UG93B as control. Primes for qRT-PCR were shown in supplementary Table S4.

\section{Statistical analysis}

Data on relative genes expression of both CMS and maintainer line as well as $\mathrm{F}_{1}$ hybrid were statistically analyzed according to the procedure appropriate for one way analysis of variance using SPSS17.0 software.

\section{CR-RT-PCR analysis}

The processing of RT-PCR analysis of circularized RNA was described by Kuhn et al. (2002). In brief, $1 \mu \mathrm{g}$ of total RNA was self-ligated with T4 RNA ligase in a total volume of $10 \mu \mathrm{L}$ under the conditions recommended by the enzyme manufacturer (Thermo Fisher Scientific, USA). First-strand synthesis was performed with primer AP1 and superscript reverse transcriptase on $1 \mu \mathrm{L}$ ligated RNA following the instructions of the respective manual (TransGene Biotech, Beijing, China). DNA fragments comprising the 5'-3' ligated mRNA end were subsequently amplified with primer pairs SP1/AP2, followed by a second PCR with nested primers SP2/AP3 using amplification products of first PCR as a template (Fig. S1). The products were finally cloned into a linearized pEASY-T1 Cloning vector according to the standard procedures followed by sequence analysis of more than 15 cDNA clones. The location of the primers was given with respect to the translation initiation codon $(+1)$. All numbering are in respect to the translation start codon $(+1)$. Primes for CR-RT-PCR analysis are shown in supplementary (Table S5).

\section{Sequence analysis}

The 5' and 3' termini of the transcripts were determined by comparison between the clone sequences and the target gene sequences using the DNAMAN software. The promoter 
architecture was described by Crooks et al (2004) and analyzed using the online software WebLogo (http://weblogo.berkeley.edu/logo.cgi).

\section{Results}

\section{Resequencing analysis of mitochondrial genome in UG93A}

mtDNA of UG93A was randomly sheared into fragments with an average length of $300 \mathrm{bp}$ to construct a paired-end sequencing library and resequenced on a HiSeq 2000 sequencing platform. Clean data were obtained after filtering low-quantity, short reads and adaptors from raw data. The number of reads, read length and total bases were counted before and after filtration. A total of $3.7 \times 10^{5}$ paired-end reads with a filtered ratio of $2.16 \%$ were obtained, as were $1 \times 10^{8}$ total bases with a filtered ratio of $11.36 \%$. Clean data were aligned to the reference mtDNA of UG93B using BWA software. The coverage of UG93A mtDNA was $99.2 \%$, aligning to UG93B mtDNA, with a coverage depth of 179× (Table S1).

\section{Analysis and annotation of SNPs in UG93A mtDNA}

A total of 398 SNPs in UG93A mtDNA with a reference mitochondrial genome sequence of UG93B were observed. The distribution rate and frequency of SNPs in the whole UG93A mtDNA were counted per unit of $50 \mathrm{kbp}$. Results showed that the frequency of UG93A mtDNA was diverse in various regions. The variable frequency of SNPs from 350-400 kb was the highest $(1.94 \mathrm{bp} / \mathrm{kb})$ with the main mutant genes of $r r n L$ and $r r n S$. Most of SNPs variation occurred in non-coding regions, intergenic regions and introns, including 362 SNPs (accounted $90 \%$ of total SNPs) distributed intergenic and introns. While only 37 SNPs (accounted 10\% of the total SNPs) distributed in the coding region of mitochondrial genes (Table S2). The amino acid annotation in the variation of the coding region was analyzed. These results showed five SNPs with synonymous mutations and thirty-two non-synonymous mutations in the gene coding region, resulting in changes of the amino residues encoded by eight mitochondrial genes. Twenty-six of these SNPs variations were found in the coding region of atp6, while only one or two SNPs among other genes were found, indicating that the coding region of atp6 in UG93A was an active recombination region (Fig. 1, Table 2). 
Table 2. Annotation of SNPs in protein-coding genes of UG93A mtDNA

\begin{tabular}{|c|c|c|c|c|c|c|}
\hline No. & $\begin{array}{c}\text { Variable } \\
\text { sites }\end{array}$ & RefSeq & $\begin{array}{c}\text { Base } \\
\text { mutation }\end{array}$ & $\begin{array}{l}\text { Gene } \\
\text { name }\end{array}$ & Amino acids & $\begin{array}{c}\text { Mutated } \\
\text { amino acids }\end{array}$ \\
\hline 1 & 92263 & $\mathbf{A}$ & $\mathbf{C}$ & rpl10 & I & $\mathbf{L}$ \\
\hline 2 & 134785 & $\mathbf{C}$ & $\mathbf{T}$ & $\operatorname{sdh} 4^{a}$ & $\mathbf{G}$ & $\mathbf{S}$ \\
\hline 3 & 192259 & $\mathrm{~A}$ & $\mathrm{G}$ & $\operatorname{coxl}$ & $\mathrm{G}$ & $\mathrm{G}$ \\
\hline 4 & 193141 & $\mathrm{C}$ & $\mathrm{T}$ & $\operatorname{cox} l$ & $\mathrm{~F}$ & $\mathrm{~F}$ \\
\hline 5 & 193750 & $\mathrm{~T}$ & G & $\operatorname{cox} l$ & $\mathrm{~L}$ & $\mathrm{~L}$ \\
\hline 6 & 193303 & $\mathrm{C}$ & A & $\operatorname{cox} l$ & I & I \\
\hline 7 & 271723 & $\mathbf{T}$ & $\mathbf{G}$ & $\operatorname{rps} 14^{a}$ & $\mathbf{L}$ & $\mathbf{F}$ \\
\hline 8 & 470838 & $\mathbf{C}$ & $\mathbf{G}$ & $\operatorname{atp}^{a}$ & $\mathbf{E}$ & D \\
\hline 9 & 470847 & $\mathbf{C}$ & $\mathbf{A}$ & atp6 & $\mathbf{E}$ & D \\
\hline 10 & 470863 & $\mathbf{C}$ & $\mathbf{T}$ & atp6 & $\mathbf{S}$ & $\mathbf{N}$ \\
\hline 11 & 470885 & $\mathbf{A}$ & $\mathbf{G}$ & atp6 & $\mathbf{Y}$ & $\mathbf{H}$ \\
\hline 12 & 470896 & $\mathbf{T}$ & $\mathbf{C}$ & atp6 & $\mathbf{K}$ & $\mathbf{R}$ \\
\hline 13 & 470911 & $\mathbf{T}$ & $\mathbf{A}$ & atp6 & $\mathbf{Q}$ & $\mathbf{L}$ \\
\hline 14 & 470927 & $\mathbf{G}$ & $\mathbf{C}$ & atp6 & $\mathbf{P}$ & $\mathbf{A}$ \\
\hline 15 & 470935 & $\mathbf{C}$ & $\mathbf{T}$ & atp6 & G & $\mathbf{E}$ \\
\hline 16 & 471116 & $\mathbf{T}$ & $\mathrm{C}$ & atp6 & $\mathbf{N}$ & D \\
\hline 17 & 471119 & $\mathbf{A}$ & $\mathbf{G}$ & atp6 & $\mathbf{F}$ & $\mathbf{L}$ \\
\hline 18 & 471128 & $\mathbf{A}$ & $\mathbf{G}$ & atp6 & $\mathbf{Y}$ & $\mathbf{H}$ \\
\hline 19 & 471131 & $\mathbf{A}$ & $\mathrm{C}$ & atp6 & $\mathbf{Y}$ & D \\
\hline 20 & 471136 & $\mathbf{C}$ & $\mathbf{A}$ & atp6 & $\mathbf{C}$ & $\mathbf{F}$ \\
\hline 21 & 471148 & $\mathbf{A}$ & G & atp6 & $\mathbf{L}$ & $\mathbf{T}$ \\
\hline 22 & 471149 & G & $\mathbf{T}$ & atp6 & $\mathbf{L}$ & $\mathbf{T}$ \\
\hline 23 & 471166 & $\mathbf{T}$ & G & atp6 & $\mathbf{E}$ & $\mathbf{S}$ \\
\hline 24 & 471167 & $\mathbf{C}$ & $\mathbf{A}$ & atp6 & $\mathbf{E}$ & $\mathbf{S}$ \\
\hline 25 & 471177 & $\mathbf{T}$ & $\mathbf{A}$ & atp6 & $\mathbf{L}$ & $\mathbf{F}$ \\
\hline 26 & 4711182 & G & $\mathbf{A}$ & atp6 & $\mathbf{H}$ & $\mathbf{Y}$ \\
\hline
\end{tabular}




\begin{tabular}{ccccccc}
\hline \multicolumn{7}{c}{ Continue } \\
\hline 27 & 471228 & A & C & atp6 & C & W \\
28 & 471232 & C & A & atp6 & G & V \\
29 & 471237 & G & T & atp6 & A & E \\
30 & 471261 & A & T & atp6 & F & L \\
31 & 471238 & G & T & atp6 & A & E \\
32 & 471273 & G & A & atp6 & S & F \\
33 & 471277 & G & A & atp6 & T & M \\
34 & 512025 & A & C & cox2 & T & T \\
35 & 512031 & G & T & cox2 & M & I \\
36 & 539731 & C & G & atp4 & I & M \\
37 & 568501 & A & T & rps $7^{a}$ & F & L \\
\hline
\end{tabular}

Boldface represent nonsynonymous mutation. a, indicated the transcription of this gene occurs on the antisense strand. 


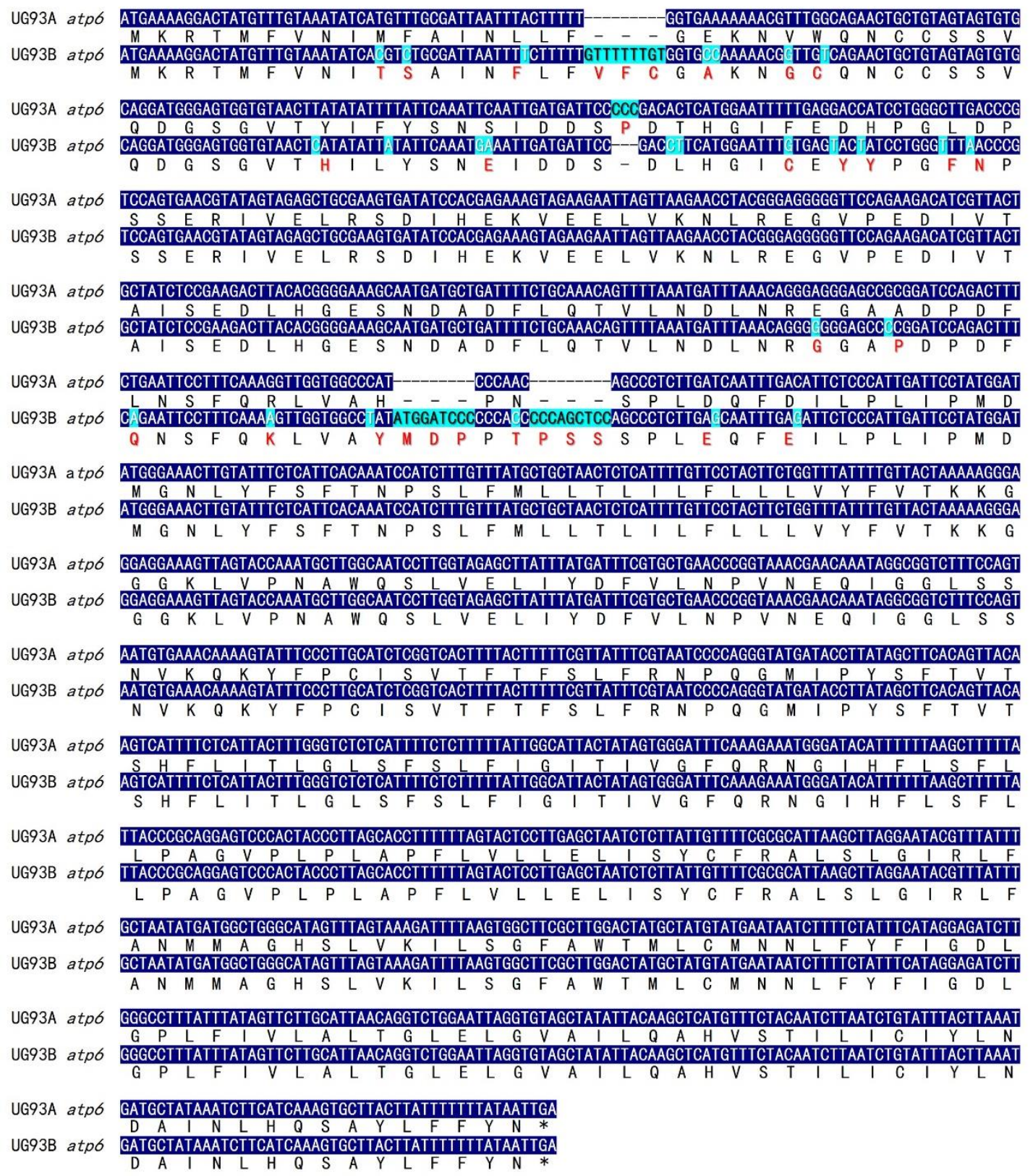

Fig. 1. Alignment of the atp6 between UG93A and UG93B. The variable nucleotide between UG93A and UG93B was marked with light blue background, and the corresponding variation of amino acid residues was indicated with red letter. 
Analysis and annotation of InDels in UG93A mtDNA

A total of 230 small fragments of insertion/deletion (InDel) variations were found in UG93A mtDNA compared to UG93B mtDNA, ranging from 1 bp to $31 \mathrm{bp}$ (Fig. 2). The short InDels were mainly distributed from $1 \mathrm{bp}$ to $5 \mathrm{bp}$ in total of 178 , accounting for $77.4 \%$ of total InDels, while 52 InDels were distributed from $6 \mathrm{bp}$ to $31 \mathrm{bp}$, accounted for 22.6\%. In particular, a total of 99 InDels with a size of 1 bp and 38 InDels with a size of 4 bp were distributed in UG93A mtDNA, accounting for $43 \%$ and $16 \%$ of the total InDels, respectively. Other InDels were rarely distributed from $2 \%$ to $6 \%$. Most InDels were distributed between the intergene region and intron. Only 11 InDels were distributed in the coding region resulting variation in amino acids encoded by mitochondrial genes (Table 1).

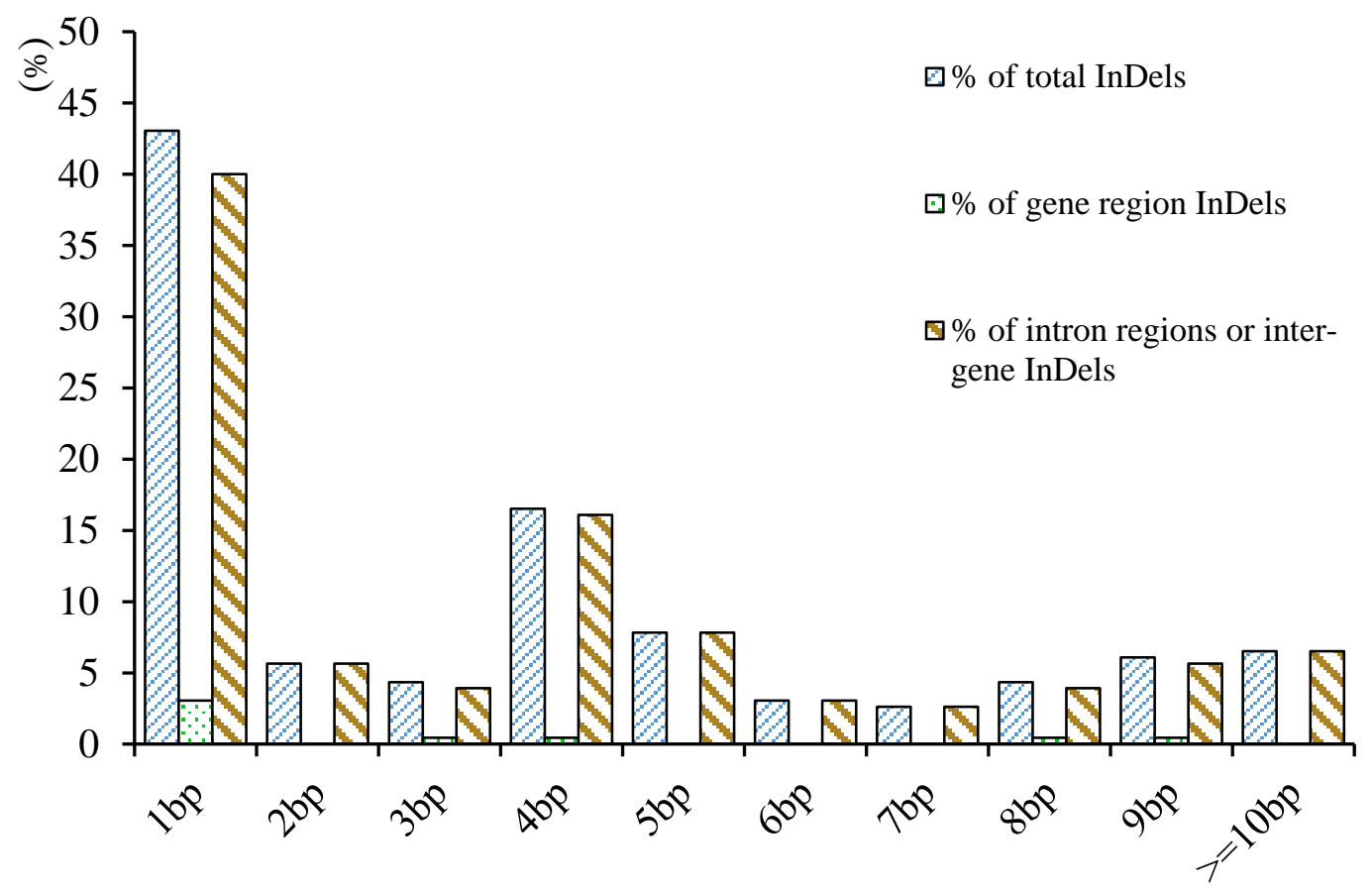

Fig. 2. Distribution of different sized InDels in UG93A mtDNA

\section{Transcript analysis of kenaf mtDNA}

To find the mitochondrial genomic regions that possibly contain the candidate genes for kenaf CMS, a total of 36 protein coding mitochondrial genes were used to 
design probe primers to examine the possible alteration in the mitochondrial transcription patterns among UG93A, UG93B and $\mathrm{F}_{1}$ (UG93A/UG93R) by Northern blotting analysis. Most of mitochondrial genes shared the same mRNA among UG93A, UG93B and $\mathrm{F}_{1}$ (UG93A/UG93R), excluding six genes, atp1, atp4, atp6, atp9, cox3 and sdh4 (Fig. 3). Moreover, these mitochondrial genes of atp1, atp4, atp6, cox3 and sdh4 showed the same transcription patterns between UG93A and $\mathrm{F}_{1}$ (UG93A/UG93R), but different from UG93B, indicating that the transcript patterns of these five mitochondrial genes were not regulated by nuclear genes in kenaf (Fig. 3). Furthermore, four probes of atpl, atp6, cox3 and $s d h 4$ detected the mRNA patterns with strong signal among UG93A, UG93B and $F_{1}$ (UG93A/UG93R), but weaker signal in UG93B with atp4 as prob (Fig. 3b). The probe of atp9 detected a special mRNA with relatively smaller size and weaker signal in UG93A and $F_{1}$ (UG93A/UG93R) (Fig. 3d), This suggests that the differential transcript of atp9 in $\mathrm{F}_{1}$ (UG93A/UG93R) may be regulated by nuclear genes despite the same cytoplasm between UG93A and $F_{1}$ (UG93A/UG93R) and correlate to kenaf CMS.

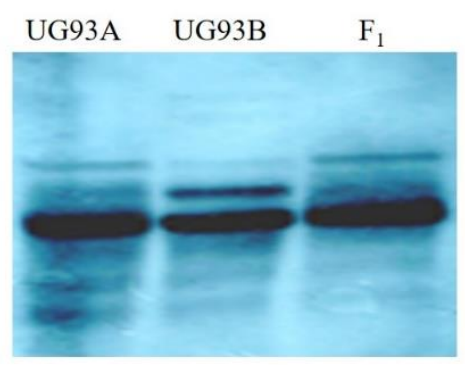

a $\operatorname{atpl}$

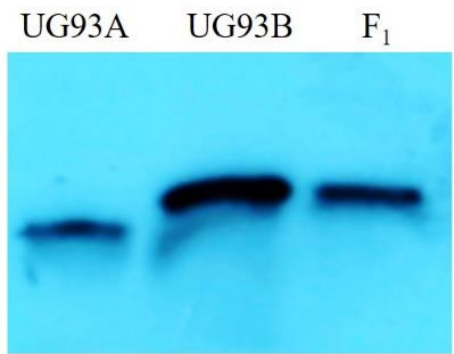

d atp 9

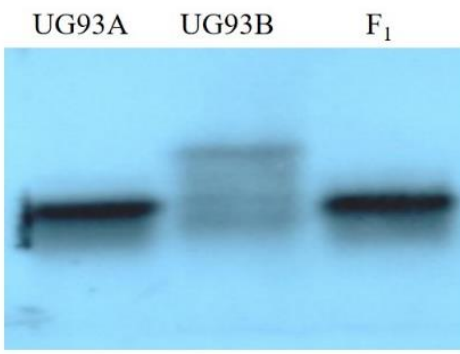

b atp4

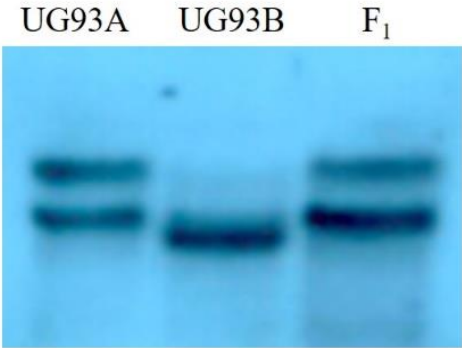

e $\operatorname{cox} 3$

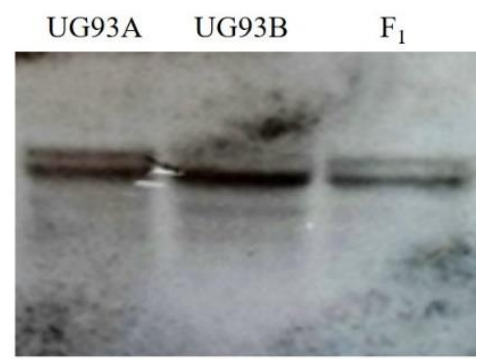

c atp6

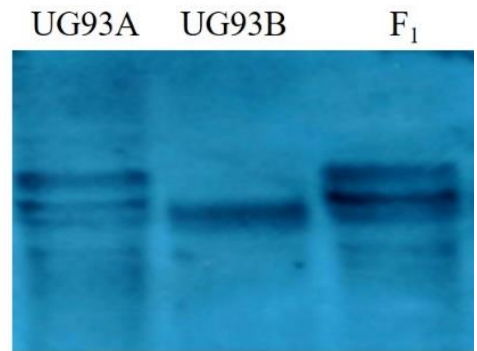

f $s d h 4$

Fig. 3. Transcriptional analysis of the kenaf mitochondrial genes. 
Real-time quantitative PCR was performed for determination of relative expression levels of atp1, atp4, atp6, atp9 and cox3 in UG93A, UG93B and $\mathrm{F}_{1}$ (UG93A/UG93R) in the monocaryotic phase and dikaryophase of kenaf anther. The levels of atpl, atp4 and atp9 in the CMS line UG93A were lower compared with UG93B in both monocaryotic phase and dikaryophase. The expression of atp9 in UG93A was significantly lower than in UG93B, indicating that atp9 played a central role in the energy supply for the microspore development in kenaf (Fig. 4a), and the relative expression in $F_{1}$ (UG93A/UG93R) was higher compared with UG93B at monocaryotic phase, while significantly lower at dikaryophase of anther development (Fig. 4a). The expression of atp6 and $\operatorname{cox} 3$ was significantly lower than UG93B and $\mathrm{F}_{1}$ (UG93A/UG93R) in the monocaryotic phase, but substantially higher than UG93B in dikaryophase (Fig. 4b). These data revealed that mitochondrial gene expression in kenaf may be regulated by multiple factors.

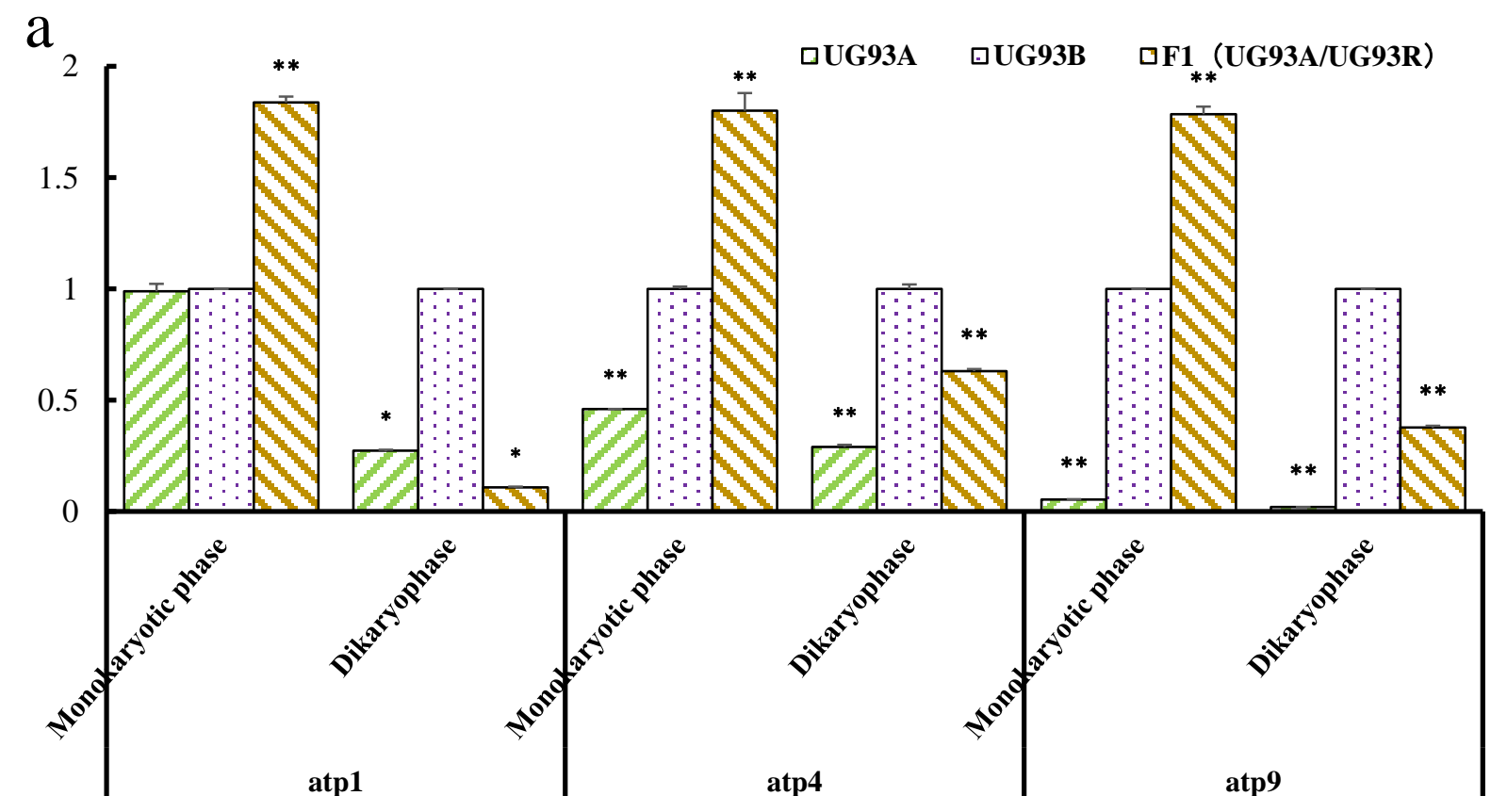




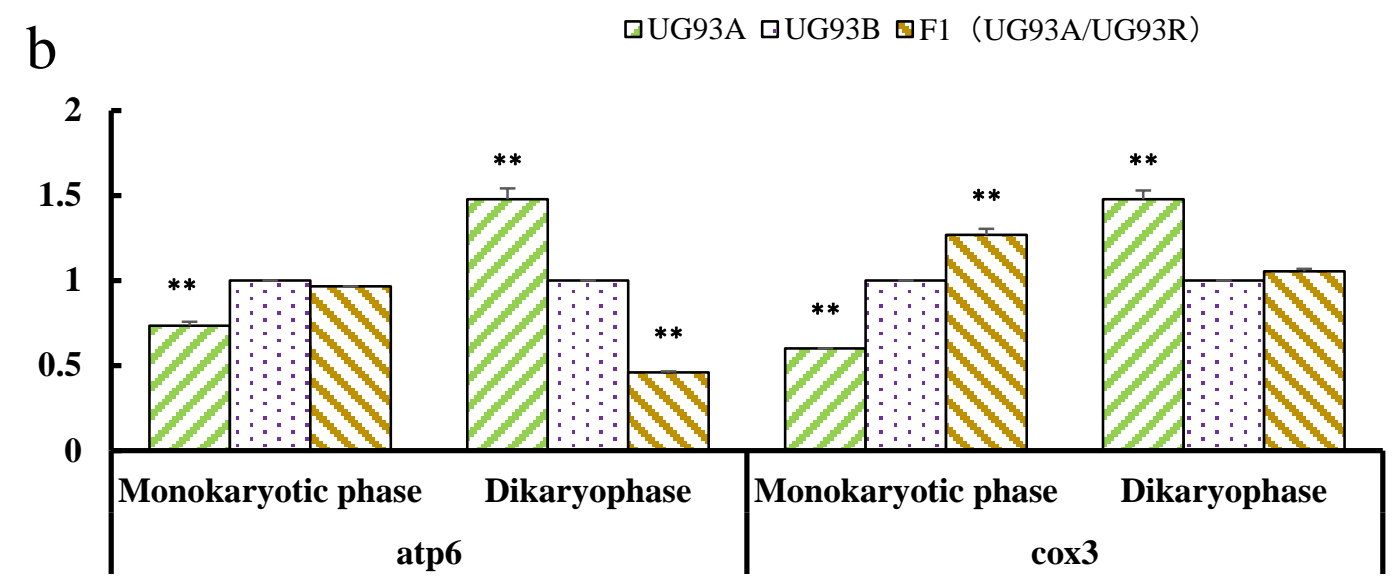

Fig. 4. Relative expression analysis of atpl, atp4, atp6, atp9 and cox3. The housekeeping gene Histone 3 was used as an internal control. Error bars represent standard deviation $(\mathrm{n}=3)$. The significance of differences was assessed by Student's t-test $(* \mathrm{P}<0.05$, $* * \mathrm{P}<0.01)$.

\section{Circularized RNA (CR)-RT-PCR analysis of mitochondrial genes}

Previous analysis revealed that the transcripts of atp1, atp4, atp6 and cox3 can be transcribed into polycistronicm transcripts, but atp 9 with monocistronic transcripts. The transcripts of atp1, atp4, atp6, atp9 and cox3 were analyzed by CR-RT-PCR in UG93A, UG93B and $F_{1}$ (UG93A/UG93R) (Fig. 5). Results showed multiple transcription initiation sites at the 5' - ends upstream of atpl, atp4, atp6 and cox3, but the transcript termination sites of $3^{\prime}$ - ends were uniform in UG93A, UG93B and $F_{1}$ (UG93A/UG93R) except for atp9. Sequences analysis revealed that the 3'- ends of atpl were uniform (+49 relative to the stop codon) in UG93A, UG93B and $F_{1}$ (UG93A/UG93R). Three 5'- ends were located at -78, -138 and -213 relative to the start codon in UG93A. Only one 5'- ends was located at -176 in UG93B and two 5'ends were located at -158 and -176 in $F_{1}$ (UG93A/UG93R). Interestingly, the CR-RT-PCR profile was different slightly with the transcript of atp1. Two major transcripts were identified in UG93B, whereas only one 5'- end was identified by CR-RT-PCR, suggesting that 5'- end could be involved in the translational efficiency 
of plant mitochondrial RNA. 2-bp insertion at the (-11) - (-12) locus and nine nucleotide variations at the position $(-92)-(-114)$ upstream of the start codon in UG93A and $F_{1}$ (UG93A/UG93R), respectively (Fig. 6, Fig S2).

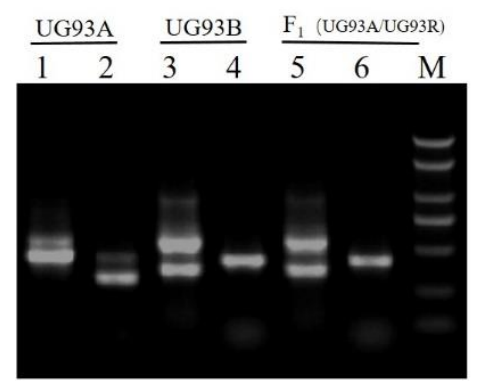

atpl

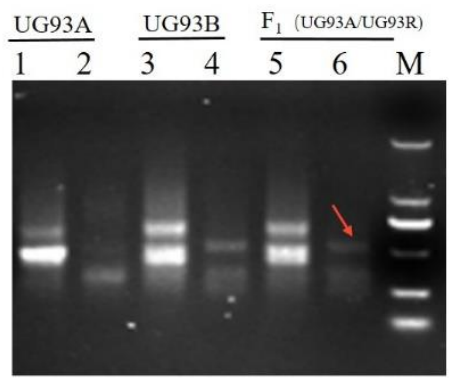

atp4

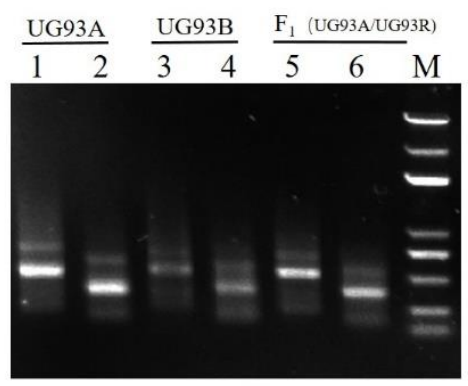

atp6

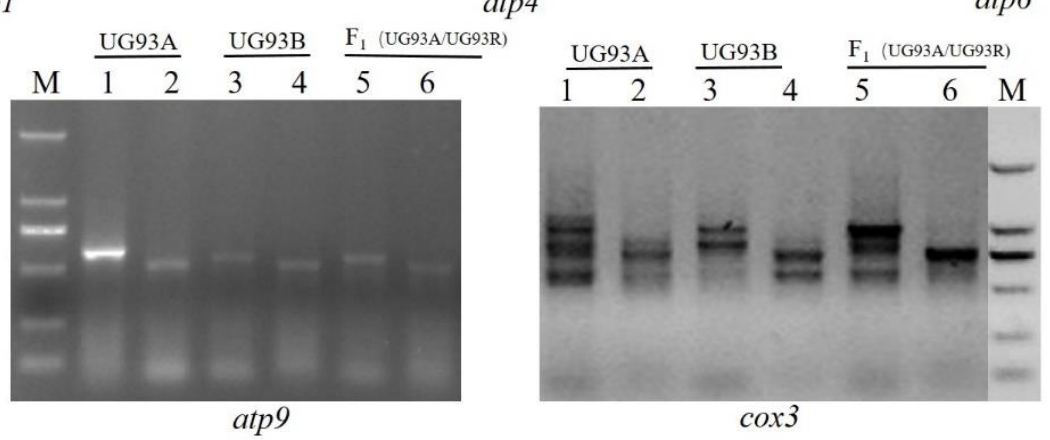

Fig. 5 CR-RT-PCR analysis of mitochondrial genes. The second amplification product of atp4 in $\mathrm{F}_{1}$ was given in red arrow.

Similar observations have been made in other mRNAs. There was only one 5'- end of atp4 located at nucleotide position -307 upstream of the start codon in UG93A. Three 5'- ends located at nucleotide positions -56, -274 and -376 upstream of the start codon in UG93B and two $5^{\prime}$ - ends located at nucleotide position -74 and -307 in $F_{1}$ (UG93A/UG93R). The 3' - ends were uniform (+ 71 relative to the stop codon) in the three cytoplasm of kenaf (Fig. S3, S4).

\begin{tabular}{|c|c|c|c|}
\hline \multirow{6}{*}{ UG93A atpl } & +49 & -78 & \multirow[b]{2}{*}{$1 / 16$} \\
\hline & TCTATCTTAAAAGAAAGCGCCTTACCTATGATG & - TATAAAAAAGAGGAG. & \\
\hline & +49 & 138 & \multirow{2}{*}{$1 / 16$} \\
\hline & TCTATCTTAAAAGAAAGCGCCTTACCTATGATGA & TCCTTATAGAAGGCT.... & \\
\hline & +49 & 213 & \multirow{2}{*}{$14 / 16$} \\
\hline & TCTATCTTAAAAGAAAGCGCCTTACCTATGATG & TGGTAGGGCATTCTC & \\
\hline \multirow{3}{*}{ UG93B atpl } & +49 & -176 & \multirow{3}{*}{$19 / 19$} \\
\hline & TCTATCTTAAAAGAAAGCGCCTTACCTATGATG & CTTTCTCAAATAGAGAA.. & \\
\hline & +49 & -158 & \\
\hline \multirow{3}{*}{$\mathrm{F}_{1}$ atpl } & TCTATCTTAAAAGAAAGCGCCTTACCTATGATG & CATGGGACCCA & \multirow[t]{2}{*}{$2 / 18$} \\
\hline & +49 & & \\
\hline & TCTATCTTAAAAGAAAGCGCCTTACCTATGATG & СТTTCTCAAATAGAGA & $16 / 18$ \\
\hline
\end{tabular}

Fig. 6. 5'- and 3'- ends detected of atpl by the CR-RT-PCR. Sequences of the cDNA 
covering the 5'-3' ligation site of atpl transcripts. The numbering of the 5'- end refers to the translation start codon and the 3'- numbers are relative to the stop codon. The numbers on the right indicate the frequency with which the distinct 5' - and $3^{\prime}$ - ends are identified in individual clones. The ligation site is indicated by a horizontal dash. Sequences corresponding to the 5'-terminal part are given in white letters in black boxes.

The transcript characteristics of atp6 in $\mathrm{F}_{1}(\mathrm{UG} 93 \mathrm{~A} / \mathrm{UG} 93 \mathrm{R})$ was identity with UG93A, located at nucleotide positions -130 and -381 upstream of the star codon, respectively, and the transcription initiation site in UG93B was located at nucleotide positions -130 and -310 upstream of the start codon, respectively. The 3' - ends were mapped at the same position in the three cytoplasm of kenaf $(+42$ relative to the stop codon) (Fig. S5). Compared with UG93B, one-bp insertion and a nine-bp deletion was identified at nucleotide position -26 and (-49)-(-57) upstream of the reading frame, respectively. Nucleotide variation happened from the position -229 upstream of the reading frame in UG93A, UG93B and $F_{1}$ (UG93A/UG93R). These results suggested that they were the active region of mitochondrial genome recombination (Fig. 7, Fig. S6). In the cDNA sequence of atp6, an RNA editing with C-T conversion occurred at the nucleotide position +1183 of reading frame in UG93A or +1204 of reading frame in UG93B, resulting in generation of a stop codon TAA, that lead to early termination of the CDS region of atp6 (Fig. 7). 


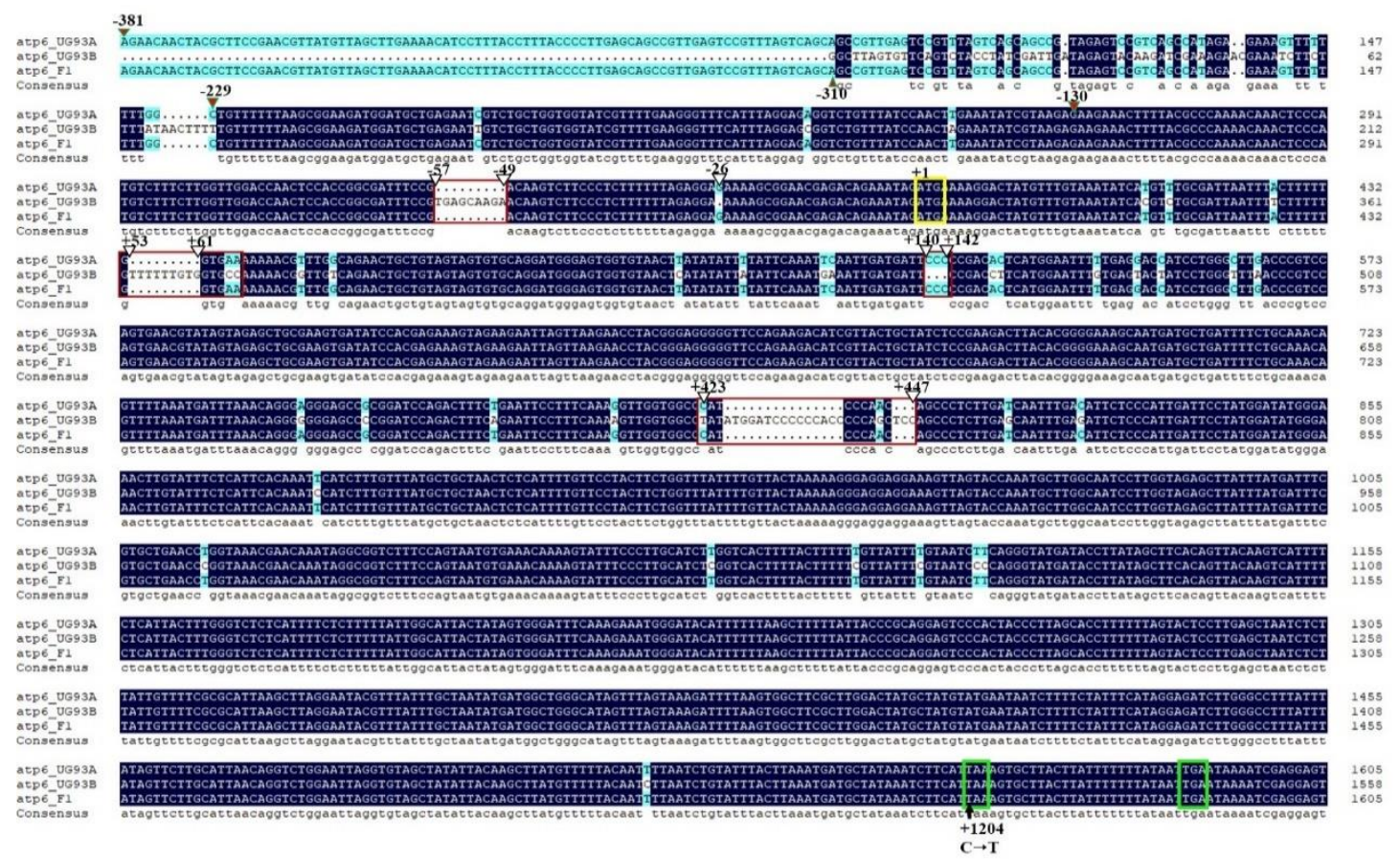

Fig. 7. Transcription sequence analysis of atp6. The initiation codon and stop codon were indicated by yellow and green box, respectively. The variable regions among UG93A, UG93B and $\mathrm{F}_{1}$ were marked with red boxes, and the variable sites were indicated by open triangles. The transcripton initiation site were indicated by solid red triangles. The C-T editing site of the cDNA sequence was indicated by black arrow.

The 5' - ends of atp9 was identity in UG93A, UG93B and $F_{1}(\mathrm{UG} 93 \mathrm{~A} / \mathrm{UG} 93 \mathrm{R})$, located at position -71 upstream of the start codon, but was different at 3'- end. The transcription termination site of the $3^{\prime}$ - end was located at +271 in UG93A, but +397 downstream of the stop codon in UG93B and $F_{1}$ (UG93A/UG93R), which was consistent with the mRNA transcript among UG93A, UG93B and $F_{1}$ (UG93A/UG93R) as previously research (Fig. S7). In the cDNA sequence of atp9, a C-T conversion was identified at the nucleotide position +262 of reading frame compared to atp 9 gDNA sequence, resulting in a generation of stop coding TGA, that lead to early termination of the CDS region of atp9 (Fig. 8). 


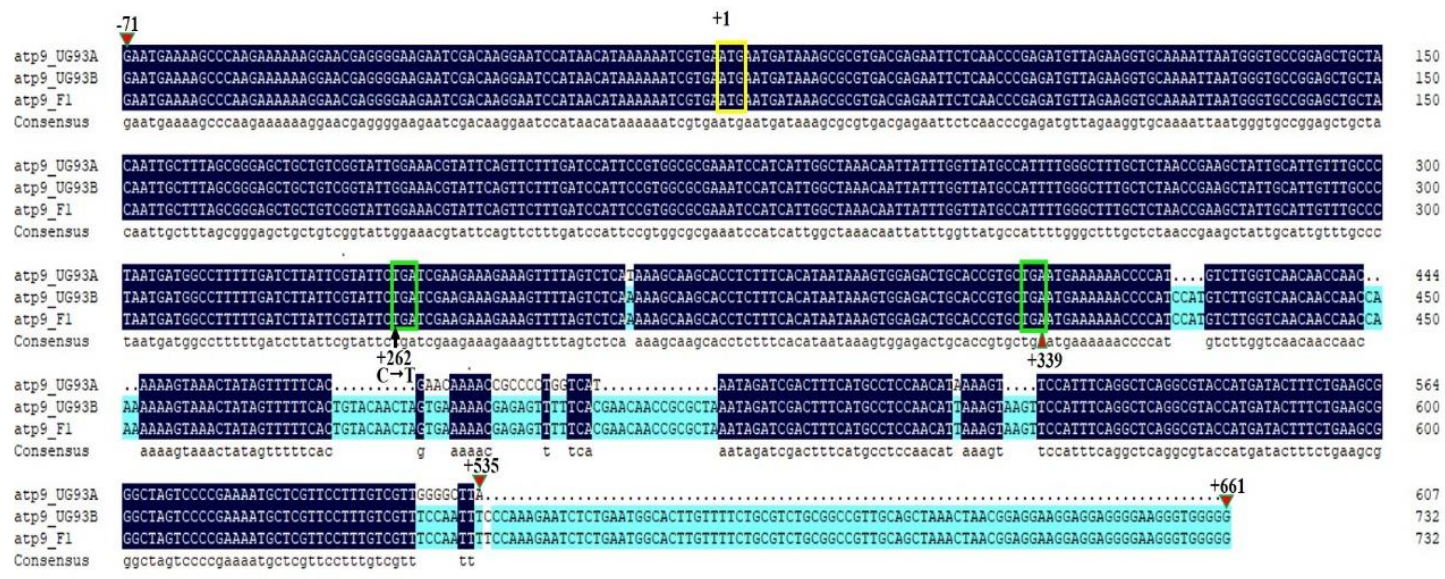

Fig. 8. Transcription analysis of atp9. The initiation codon and stop codon were indicated by yellow and green box, respectively. The variable sites were indicated by solid red triangle.

The cox3 RNA was shown to accumulate in two different forms with the 5'- ends located at -164 and -313 upstream of the start codon in UG93A. One 5'- ends mapped at the -311 in UG93B, and two 5'- ends located at nucleotide positions -313 and -442 upstream of the start codon. The 3' - ends were mapped at the same position in the three cytoplasmic of kenaf (+314 relative to the stop codon) (Fig. S8, S9).

\section{The highly diverged of the mitochondrial promoter architecture in kenaf}

The YRTA motifs are widely present in the mitochondrial promoters of monocot and dicot plant species. A search for analyzing the consensus sequences in kenaf mitochondrial promoter regions found that only eleven of them contained the YRTA motifs (Table 2.). Six of these core element sequences are located at the position of -4 to -7 upstream of the transcriptional start, and the other five are located at the position of -25 to -29 upstream of the transcriptional start. These promotor sequences were further analyzed with WebLogo software, which is designed to generate sequence logos within a multi-sequence alignment for sequence conservation at particular positions. Our result showed that the promoter sequences were less conserved overall, but the nucleotide of $\mathrm{T}$ or A were observed with highly frequency at the upstream of initiation codon (Fig. 9). Our data suggest that the promoter sequences in kenaf mitochondrial genome are highly diverged, and the YRTA motif is not an essential element for the kenaf mitochondrial promoter activity. 


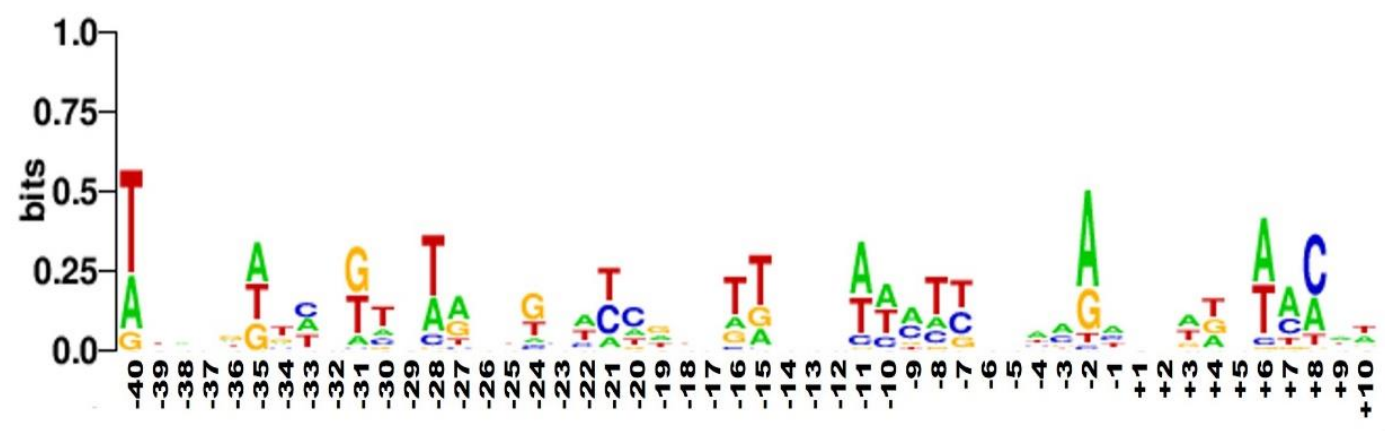

Fig. 9. Summary of nucleotide sequences around the defined transcription initiation sites in kenaf mitochondrial genes (displayed in Table 2). Position +1 corresponds to the transcription initiation sites. The overall height of each stack indicates the sequence conservation at that position (measured in bits), whereas the height of each nucleotide within the stack reflects its relative frequency among the sequences. 
Table 2 The transcription initiation sites and flanking sequences of the mitochondrial transcripts in kenaf.

\begin{tabular}{|c|c|c|c|}
\hline Gene & $\begin{array}{c}\text { Transcription initiation } \\
\text { site relative to the stat } \\
\text { codon }\end{array}$ & Sequence around transcription initiation site & No. clone sequenced \\
\hline \multirow{3}{*}{ atp1-UG93A } & -78 & ATGCTATTTATAATAAAGAGTTATTTATTGACTTTGATGGTATAAAAAAG & $1 / 16$ \\
\hline & -138 & 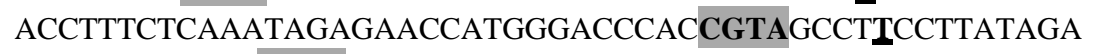 & $1 / 16$ \\
\hline & -213 & AACAAAGGCACCATATTAGGGGGTTCCTATTCTATCCTAATGGTAGGGCA & $14 / 16$ \\
\hline atp1-UG93B & -176 & TAATGGTAGGGCATTCTCATTGGATACCTACAACACCCACㅌTTCTCAAA & $19 / 19$ \\
\hline \multirow{2}{*}{ atp1-F1 } & -158 & ATTGGATACCTACAACACCCACCTTTCTCAAATAGAGAAC息ATGGGACCC & $2 / 18$ \\
\hline & -176 & TAATGGTAGGGCATTCTCATTGGATACCTACAACACCCACㅡㅡTTCTCAAA & $16 / 18$ \\
\hline atp4-UG93A & -307 & GGTTTAGTATCAAGGTGATTCTGTTTCTGTTCCTATATAT音TGGGTCCGT & $15 / 15$ \\
\hline \multirow{2}{*}{ atp4-UG93B } & -74 & TTCTTTCGATAGCTATTTTTGGTTCCGTTAGAGCTGTAAACGTTTTTCAC & $2 / 15$ \\
\hline & -307 & 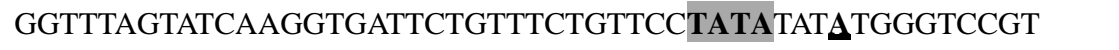 & $8 / 15$ \\
\hline \multirow{3}{*}{ atp4-F1 } & -56 & TTGGTTCCGTTAGAGCTGTAAACGTTTTTCACTACTAGA音GTCTCTCCT & $5 / 15$ \\
\hline & -274 & TCAATTAATGGGACTTGGCTGGAAAGTGTTCTTGCCTCTATCATTAGCTT & $5 / 15$ \\
\hline & -376 & 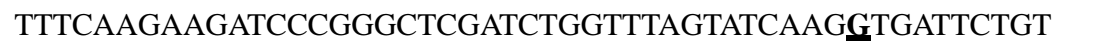 & $10 / 15$ \\
\hline \multirow{2}{*}{ atp6-UG93A } & -122 & TTAGGAGAGGTCTGTTTATCCAACTTGAAATATCGTAAGA-GAAGAAACTT & $12 / 15$ \\
\hline & -381 & TTTCAGACCTCTTAGACCCCAAGCAAGAAATATCGTACATÄGAACAACTA & $3 / 15$ \\
\hline \multirow{2}{*}{ atp6-UG93B } & -130 & 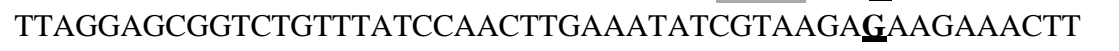 & $13 / 17$ \\
\hline & -310 & GAACGAACGAATGGATTTTTTTTGTTTCTTTTTCGAGATTEGGCTTAGTGT & $4 / 17$ \\
\hline \multirow{2}{*}{ atp6-F1 } & -122 & TTAGGAGAGGTCTGTTTATCCAACTTGAAATATCGTAAGAGAAGAAACTT & $10 / 15$ \\
\hline & -381 & TTTCAGACCTCTTAGACCCCAAGCAAGAAATATCGTACAT & $5 / 15$ \\
\hline atp9- UG93A & -71 & TTGGTGTTCAGTGTACCGCACCGTGGGTACAAGATCGAAAAGAATGAAAAG & $15 / 15$ \\
\hline atp9- UG93B & -71 & 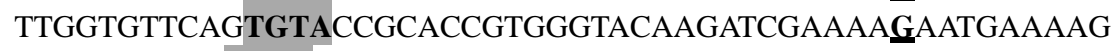 & $15 / 15$ \\
\hline atp9- F1 & -71 & 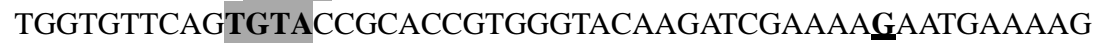 & $15 / 15$ \\
\hline \multirow{2}{*}{ cox3-UG93A } & -164 & TGGTCTCTGGTTTGATGGTTGGTGTGGTCCATTTTCCCACÉTAGTTCGTC & $4 / 15$ \\
\hline & -313 & ACAAAGTTTTTCTAGAGCAACTTCTTAGGTACTGGCTGAC드ATTACAA & $11 / 15$ \\
\hline cox3-UG93B & -313 & ACAAAGTTTTTCTAGAGCAACTTCTTAGGTACTGGCTGACT̄CTATTACAA & $15 / 15$ \\
\hline \multirow[t]{2}{*}{$\operatorname{cox} 3-\mathrm{F} 1$} & -313 & ACAAAGTTTTTCTAGAGCAACTTCTTAGGTACTGGCTGACTCTATTACAA & $2 / 15$ \\
\hline & -442 & ATCGCTTAATCCAAATATTAAGCGAATCCCCCTTTCCCGA프AGTGCCATA & $13 / 15$ \\
\hline
\end{tabular}

The transcription initiation nucleotides are underlined with bold, and the promoter cores of YRTA (Y=T or C, R=A or G) motifs are written with bold and highlighted. 


\section{Discussion}

Comparative analysis of UG93A and UG93B mtDNAs

The evolution of the plant mitochondrial genome primarily includes genomic repeats, rearrangement, insertions, deletions, and nucleotide mutations among genomes (Clifton et al., 2004; Tian et al., 2006; Darracq et al., 2010). In present study, a total of 398 SNPs and 230 InDels in UG93A compared to those in the reference mitochondrial genomic UG93B, far lower than those in Brassica napus and rice, which conserved 36,458 and 76,370 variations, respectively (Huang, 2013; Wang et al., 2013). Since both CMS line UG93A and maintainer line UG93B were bred from wild-type UG93, belonging to cytoplasmic near-isogenic line. This line can be effectively eliminated the interference of redundant genetic information irrelevant to kenaf CMS. Hence these are ideal materials for understanding the molecular mechanism of kenaf cytoplasmic male sterility.

Plant mitochondrial genome is labile due to its recombination frequently among mitochondrial, chloroplast and nuclear DNA (Mower et al., 2012). But most of the recombination sequences are located in intergenic regions and show a faster rate of evolution (Allen et al., 2007). Furthermore, although many gene sequences were highly conserved between UG93A and UG93B, there were exceptions. The sequences of rpl10, sdh4, cox1, rps14, cox2, atp4, rps7 between UG93A and UG93B were different, especially atp6 that conserve active recombination region between UG93A and UG93B, which could be a good candidate genes for contributing to kenaf CMS phenotype. Finally, the number of SNPs between UG93A and UG93B mtDNA was aslo significant compared to those in a CMS line of sugar beet, which has 24 SNPs in 11 protein-coding genes compared to the fertile line (Satoh et al., 2004).

SNPs and InDels are important factors that are responsible for biodiversity and are widely found in genome of plants and animals (Feltus et al., 2004; Shen et al., 2004; Mills et al., 2006). In present study, most SNP and InDel variants occurred in non-coding regions, intergenic regions and introns of UG93A mtDNA and the distribution of InDels were mainly from 1 bp to 5 bp as identified in rice (Wang et al., 
2013) and Chinese cabbage (Park et al., 2010). As InDels in coding region or promoter region are harmful to human health thus would be eliminated, while InDel variations between introns and intergenes need to be retained (Mills et al., 2011). This suggested that variations in SNPs and InDels of genome could act a self-protection mechanism in process of biological evolution.

\section{Expression of mitochondrial genes in kenaf CMS}

It has been documented that an altered transcription profile in CMS lines comparing to their maintainer lines and fertility-restored plants (hybrids) is one of the main features observed for CMS-associated genes (Hanson and Bentolila, 2004; Luo et al., 2013). Many CMS genes have been proved to contain sequences similar to mitochondrial respiratory chain complex genes and co-transcribed with these genes, such as atp1, atp6, atp8, atp9 and cox2 (Hanson and Bentolila, 2004). In this study, we examined the transcript profile among CMS line UG93A, maintainer line UG93B and hybrid $F_{1}$ by using of the 36 protein coding mitochondrial genes as RNA blot probes. A specific mRNA with atp9 as probe was detected in UG93A, which has a smaller transcript size as compare to $\mathrm{UG} 93 \mathrm{~B}$ and $\mathrm{F}_{1}$ and distinct from other plants that CMS genes co-transcribed with adjacent essential genes to produce larger, dicistronic mRNAs (Liu et al., 2007; Wang et al., 2013). This data suggesting that it could be a novel regulation model to kenaf CMS, sequencing and functional analysis of atp9 region is underway.

In present study, expression of atpl in UG93A and $\mathrm{F}_{1}$ (UG93A/UG93R) was lower compared with UG93B in the dicaryotic phase of anther development. Unlike expression of atpl in isonuclear alloplasmic CMS line P3A, P3B and $\mathrm{F}_{1}(\mathrm{P} 3 \mathrm{~A} / 992)$, which was lower than $\mathrm{P} 3 \mathrm{~B}$ and $\mathrm{F}_{1}(\mathrm{P} 3 \mathrm{~A} / 992)$. These current findings indicated that expression of atpl were different in various cytoplasms, probably due to inconsistent nuclear background. The expression of atp6 was lower in CMS line and $\mathrm{F}_{1}$ (UG93A/UG93R) compared with UG93B and consistent with expression of atp6 in $\mathrm{P} 3 \mathrm{~A}$, which was downward than in $\mathrm{P} 3 \mathrm{~B}$ and $\mathrm{F}_{1}(\mathrm{P} 3 \mathrm{~A} / 992)$ (Zhao et al., 2016). This 
showed that atp6 may be involved post-transcription in the kenaf CMS line. The expression of atp9 in UG93A was significantly lower than in UG93B and $\mathrm{F}_{1}$ (UG93A/UG93R), revealing its significance in development of microspores of kenaf. While the expression of atp9 in ramee male sterile line was significantly higher than fertile line (Duan et al., 2008), indicating that the regulation of atp9 was different among various crops. However, the expression of atp4 in UG93B was higher, which was contrary to transcript that had weaker signal comparing with UG93A, suggesting that the expression of atp 4 was regulated by post-transcriptional processing. In brief, the expression of kenaf mitochondrial genes was significant difference in various cytoplasms, suggesting that pollen abortion in kenaf was resulted from differential expression of subunit gene in mitochondrial respiratory chain complex, leading in a insufficient energy supplying to pollen development.

\section{Heterogeneity of the 5'- end in plant mitochondrial genes}

Multiple promoters are common feature of mitochondrial genes in plants (Kuhn et al., 2005). A total of 30 transcription initiation sites were found in 12 mitochondrial genes in Arabidopsis, while only one termination site located at the 3' - end (Forner et al., 2007). There were also multiple transcription initiation sites located upstream of atpl, atp6 and atp8 while only one termination site located at the 3' - end in rice (Kazama et al., 2013; Wang et al., 2013). In this study, we found only one transcription termination site located downstream of atpl, atp4, atp6 and cox3 in various cytoplasms of kenaf, but multiple transcription initiation sites located at the 5' - end, resulting in different lengths of 5' UTR. This may be associated with translation of mitochondrial genes and regulated by nuclear genes (Koizuka et al., 2003; Kazama et al., 2008), as the RNA processing factor RPF2 having triangular pentapeptide repeat required for the 5' - mRNA formation of higher plant mitochondria (Jonietz et al., 2010). The initiation site at 5'- end of atp9 was identical in UG93A, UG93B and $F_{1}$ (UG93A/ UG93R), but 3' - end was different, having 126-bp shortage in UG93A compared with UG93B and $F_{1}$ (UG93A/ UG93R). The present results was consistent 
with the 5' -transcription initiation site of rps3, which was same in both sterile cytoplasm and fertile cytoplasm of sugar beet having 460-bp shortage at the $3^{\prime}$ transcription termination end of sterile cytoplasm compared to fertile cytoplasm (Matsunaga et al., 2011).

Our analysis on the promoter architecture showed that most of the kenaf mitochondrial promoters were not conserved with the YRTA motifs. This is fairly differ from that in other dicot plants, which showed immediately upstream a CRTA core sequence (Kuhn et al., 2005). The results showed that that the promoter sequences in kenaf mitochondrial genome are more diverged than those in other plant species, indicating that the YRTA motif is not an essential element for the kenaf mitochondrial promoter activity. However, A or T-rich elements immediately upstream of the promoters were present, which is similar to those observed in other plant mitochondrial gene promoters that have been shown to be essential for the function of the promoters (Zhang and Liu, 2006). Our data highlighted substantial diversity in transcriptional regulation of mitochondrial genes, and the molecular mechanism in regulation of mitochondrial gene transcription need further research for better understanding and confirmation in light of current findings.

\section{Conclusion}

Understanding the molecular mechanism of CMS in kenaf is important for heterosis. Plant CMS is closely related to rearrangement and transcriptional regulation of its mitochondrial genome. Comparative genome and Northern blot shows that the CDS of atp6 had active region of mitochondrial genome recombination and the transcript of atp 9 in $\mathrm{F}_{1}$ was regulated by nuclear genes, despite having the same cytoplasm with UG93A. The qRT-PCR showed that expression of kenaf mitochondrial genes was significant difference in various cytoplasms, suggesting that pollen abortion was resulted from differential expression of subunit gene in mitochondrial respiratory chain complex, leading in insufficient energy supplying to pollen development. CR-RT-PCR showed that there were multiple transcription initiation sites at the $5^{\prime}$ - 
end of mitochondrial genes, revealing common feature of the 5'- end heterogeneity of mitochondrial genes in kenaf. Our result has provided the basic information for elucidating the mechanism of CMS in kenaf and hence can be used as theoretical reference for better understanding the transcriptional regulation of mitochondrial genes.

\section{Acknowledgments}

This study was supported by the National Science Foundation of China (No. 31571719 and No. 31660430), the Chinese Postdoctoral Science Foundation (No. 2016M592608) and Natural Science Foundation of Guangxi Province (No. 2018JJB130045). The authors thank the Wuhan NextOmics Biotechnology Limited Company (China) for their help in resequencing the UG93A mtDNA of kenaf.

\section{Author Contributions}

R. Z. initiated the experiment. X. L. conducted the experiment and drafted the manuscript. X. K. and B. Z. isolated the kenaf mtDNA. M. L. conducted the Northern blot analysis. M. W. and S. P. assisted with the experiment. A. K. and F. M. revised the manuscript and Y. Z. provided suggestions and edits for the manuscript.

\section{References}

Adams KL, Qiu YL, Stoutemyer M. and Palmer JD. 2002. Punctuated evolution of mitochondrial gene content: high and variable rates of mitochondrial gene loss and transfer to the nucleus during angiosperm evolution. Proc Natl Acad Sci U S A 99, 9905-9912.

Allen JO, Fauron CM, Minx P, et al. 2007. Comparisons among two fertile and three male-sterile mitochondrial genomes of maize. Genetics 177, 1173-1192.

Alverson, AJ, Wei X, Rice DW, Stern, DB, Barry K. and Palmer JD. 2010. Insights into the evolution of mitochondrial genome size from complete sequences of Citrullus lanatus and Cucurbita pepo (Cucurbitaceae.) Mol Biol Evol 27, 1436-1448.

Chen L, Liu YG. 2014. Male sterility and fertility restoration in crops. Annual Review of Plant 
Biology 65, 579-606.

Clifton SW, Minx P, Fauron CM, et al. 2004. Sequence and comparative analysis of the maize NB mitochondrial genome. Plant Physiology 136, 3486-3503.

Cox MP, Peterson DA, Biggs PJ. 2010. SolexaQA: At-a-glance quality assessment of Illumina second-generation sequencing data. BMC Bioinformatics 11, 485.

Crooks GE, Hon G, Chandonia JM, Brenner SE. 2004. WebLogo: A sequence logo generator. Genome Research, 14:1188-1190.

Darracq A, Varre JS, Marechal-Drouard L, et al. 2011. Structural and content diversity of mitochondrial genome in beet: a comparative genomic analysis. Genome Biology and Evolution 3, 723-736.

Duan JQ, Du GH, Li JY, Liang XN, Liu FH. 2008. Cloning and expression of atp6 and atp9 genes from ramie (Boehmeria nivea (L.) Gaud.) and their relationship with cytoplasmic male sterility. Yi Chuan 30, 1487-1498 (in Chinese).

Feltus FA, Wan J, Schulze SR, Estill JC, Jiang N, Paterson AH. 2004. An SNP resource for rice genetics and breeding based on subspecies indica and japonica genome alignments. Genome Research 14, 1812-1819.

Forner J, Weber B, Thuss S, Wildum S, Binder S. 2007. Mapping of mitochondrial mRNA termini in Arabidopsis thaliana: t-elements contribute to 5' and 3' end formation. Nucleic Acids Research 35, 3676-3692.

Fujii S, Kazama T, Yamada M, Toriyama K. 2010. Discovery of global genomic re-organization based on comparison of two newly sequenced rice mitochondrial genomes with cytoplasmic male sterility-related genes. BMC Genomics 11, 209.

Hanson MR, Bentolila S. 2004. Interactions of mitochondrial and nuclear genes that affect male gametophyte development. Plant Cell 16 Suppl, S154-S169

Heng S, Gao J, Wei C, et al. 2018. Transcript levels of orf288 are associated with the hau cytoplasmic male sterility system and altered nuclear gene expression in Brassica juncea. Journal of Experimental Botany 69, 455-466.

Heng S, Wei C, Jing B, Wan Z, Wen J, Yi B, Ma C, Tu J, Fu T, Shen J. 2014. Comparative analysis of mitochondrial genomes between the hau cytoplasmic male sterility (CMS) line and 
its iso-nuclear maintainer line in Brassica juncea to reveal the origin of the CMS-associated gene orf288. BMC Genomics 15, 322.

Huang S, Deng L, Guan M, Li J, Lu K, Wang H, Fu D, Mason AS, Liu S, Hua W. 2013. Identification of genome-wide single nucleotide polymorphisms in allopolyploid crop Brassica napus. BMC Genomics 14, 717.

Jing B, Heng S, Tong D, Wan Z, Fu T, Tu J, Ma C, Yi B, Wen J, Shen J. 2012. A male sterility-associated cytotoxic protein ORF288 in Brassica juncea causes aborted pollen development. Journal of Experimental Botany 63, 1285-1295.

Jonietz C, Forner J, Holzle A, Thuss S, Binder S. 2010. RNA processing factor2 is required for 5' end processing of nad9 and cox3 mRNAs in mitochondria of Arabidopsis thaliana. Plant Cell 22, 443-453.

Kazama T, Nakamura T, Watanabe M, Sugita M, Toriyama K. 2008. Suppression mechanism of mitochondrial ORF79 accumulation by $R f 1$ protein in BT-type cytoplasmic male sterile rice. Plant Journal 55, 619-628.

Kazama T, Yagi Y, Toriyama K, Nakamura T. 2013. Heterogeneity of the 5'- end in plant mRNA may be involved in mitochondrial translation. Frontiers in Plant Science 4, 517.

Koizuka N, Imai R, Fujimoto H, Hayakawa T, Kimura Y, Kohno-Murase J, Sakai T, Kawasaki S, Imamura J. 2003. Genetic characterization of a pentatricopeptide repeat protein gene, orf687, that restores fertility in the cytoplasmic male-sterile Kosena radish. Plant Journal 34, 407-415.

Kubo T, Nishizawa S, Sugawara A, Itchoda N, Estiati A, Mikami T. 2000. The complete nucleotide sequence of the mitochondrial genome of sugar beet (Beta vulgaris L.) reveals a novel gene for tRNA(Cys)(GCA). Nucleic Acids Research 28, 2571-2576.

Kuhn J, Binder S. 2002. RT-PCR analysis of 5' to 3'-end-ligated mRNAs identifies the extremities of cox2 transcripts in pea mitochondria. Nucleic Acids Research 30, 439-446.

Kuhn J, Tengler U, Binder S. 2001. Transcript lifetime is balanced between stabilizing stem-loop structures and degradation-promoting polyadenylation in plant mitochondria. Molecular Cell Biology 21, 731-742.

Kuhn K, Weihe A, Borner T. 2005. Multiple promoters are a common feature of mitochondrial 
genes in Arabidopsis. Nucleic Acids Research 33, 337-346.

Li H, Durbin R. 2009. Fast and accurate short read alignment with Burrows-Wheeler transform, Bioinformatics 25, 1754-1760.

Liao X, Zhao, Y, Chen P, Zhou B, Diao Y, Yu M, Huang Z, Zhou R. 2016. A comparative analysis of the atp 8 gene between a cytoplasmic male sterile line and its maintainer and further development of a molecular marker specific to male sterile cytoplasm in kenaf (Hibiscus cannabinus L.). Plant Molecular Biology Reporter 34, 29-36.

Liao X, Zhao Y, Kong X, Khan A, Zhou B, Liu D, Kashif MH, Chen P, Wang H, Zhou R. 2018. Complete sequence of kenaf (Hibiscus cannabinus) mitochondrial genome and comparative analysis with the mitochondrial genomes of other plants. Scientific Reports $\mathbf{8}$, 12714

Liu H, Cui P, Zhan K, et al. 2011. Comparative analysis of mitochondrial genomes between a wheat K-type cytoplasmic male sterility (CMS) line and its maintainer line. BMC Genomics 12, 163.

Liu Y, He X. 2006. Extraction of total RNA from cotton (Gossypium hirsutum L.) tissues with CTAB-PVP method. Journal of China Agricultural University 1, 53-56 (in Chinese).

Liu Z, Guo J, Liu Y, Xu H,. 2007. Structural and expressional variations of the mitochondrial genome conferring the wild abortive type of cytoplasmic male sterility in rice. Journal of Integrative Plant Biology 49, 908-914.

Luo D, Xu H, Liu, Z, et al. 2013. A detrimental mitochondrial-nuclear interaction causes cytoplasmic male sterility in rice. Nature Genetic 45, 573-577.

Manavski N, Guyon V, Meurer J, Wienand U, Brettschneider R. 2012. An essential pentatricopeptide repeat protein facilitates 5 ' maturation and translation initiation of $r p s 3$ mRNA in maize mitochondria. Plant Cell 24, 3087-3105.

Matsunaga M, Nagano H, Mikami T, Kubo T. 2011. Large 3' UTR of sugar beet $r p s 3$ is truncated in cytoplasmic male-sterile mitochondria. Plant Cell Reports 30, 231-238.

Mills RE, Luttig CT, Larkins CE, Beauchamp A, Tsui C, Pittard WS, Devine SE. 2006. An initial map of insertion and deletion (INDEL) variation in the human genome. Genome Research 16, 1182-1190. 
Mills RE, Pittard WS, Mullaney JM, et al. 2011. Natural genetic variation caused by small insertions and deletions in the human genome. Genome Research 21, 830-839.

Monti A, Alexopoulou E. 2013. Kenaf: a multi-purpose crop for several industrial applications. London, UK: Springer-Verlag London, 105-143.

Mower JP, Sloan DB, Alverson AJ. 2012. Plant Mitochondrial Genome Diversity: The Genomics Revolution. Vienna: Springer, Plant Genome Diversity Volume 1, 123-144.

Okazaki M, Kazama T, Murata H, Motomura K, Toriyama K. 2013. Whole mitochondrial genome sequencing and transcriptional analysis to uncover an RT102-type cytoplasmic male sterility-associated candidate Gene Derived from Oryza rufipogon. Plant and Cell Physiology 54, 1560-1568.

Park S, Yu HJ, Mun JH, Lee SC. 2010. Genome-wide discovery of DNA polymorphism in Brassica rapa. Molecular Genetics and Genomics 283, 135-145.

Porebski S, Bailey LG, Baum BR. 1997. Modification of a CTAB DNA extraction protocol for plants containing high polysaccharide and polyphenol components. Plant Molecular Biology Reporter 15, 8-15.

Reddemann A, Horn R. 2018. Recombination events involving the atp9 gene are associated with male sterility of CMS PET2 in sunflower. International Journal of Molecular Sciences 19, 806

Rodriguez-Moreno L, Gonzalez VM, Benjak A, Marti MC, Puigdomenech P, Aranda MA, Garcia-Mas J. 2011. Determination of the melon chloroplast and mitochondrial genome sequences reveals that the largest reported mitochondrial genome in plants contains a significant amount of DNA having a nuclear origin. BMC Genomics 12, 424.

Satoh M, Kubo T, Nishizawa S, Estiati A, Itchoda N, Mikami T. 2004. The cytoplasmic male-sterile type and normal type mitochondrial genomes of sugar beet share the same complement of genes of known function but differ in the content of expressed ORFs. Molecular genetics and genomics 272, 247-256.

Shen YJ, Jiang H, Jin JP, et al. 2004. Development of genome-wide DNA polymorphism database for map-based cloning of rice genes. Plant Physiology 135, 1198-1205.

Sloan DB, Alverson AJ, Chuckalovcak JP, Wu M, McCauley DE, Palmer JD, Taylor DR. 2012. Rapid evolution of enormous, multichromosomal genomes in flowering plant 
mitochondria with exceptionally high mutation rates. Plos Biology 10, e1001241.

Tao AF, Qi JM, Lin PQ, Fang PP, Wu JM, Lin LH. 2008. Cluster Analysis and Evaluation of Elite Kanaf Germplasm. Scientia Agricultura Sinica 41, 2859-2867(in Chinese).

Tian X, Zheng J, Hu S, Yu J. 2006. The rice mitochondrial genomes and their variations. Plant Physiology 140, 401-410.

Uyttewaal M, Mireau H, Rurek M, Hammani K, Arnal N, Quadrado M, Giege P. 2008. PPR336 is associated with polysomes in plant mitochondria. Journal of Molecular Biology 375, 626-636.

Wang J, Wang X, Xu H, Tang H, Zhang G, Liu Y. 2013. Structural and Expressional Variation Analyses of Mitochondrial Genomes Reveal Candidate Transcripts for the $\mathrm{S}^{\mathrm{V}}$ Cytoplasmic Male Sterility in Wheat (Triticum aestivum L.). Journal of Genetics and Genomics 40, 437-439.

Wang K, Gao F, Ji Y, Liu Y, Dan Z, Yang P, Zhu Y, Li S. 2013. ORFH79 impairs mitochondrial function via interaction with a subunit of electron transport chain complex III in Honglian cytoplasmic male sterile rice. New Phytologist 198, 408-418.

Wang ZH, Zhang D, Bai Y, et al. 2013. Genomewide variation in an introgression line of rice-Zizania revealed by whole-genome re-sequencing. PLoS One 8, e74479.

Wilson AJ, Chourey PS. 1984. A rapid inexpensive method for the isolation of restrictable mitochondrial DNA from various plant sources. Plant Cell Reports 3, 237-239.

Zhang QY, Liu YG. 2006. Rice mitochondrial genes are transcribed by multiple promoters that are highly diverged. Journal of Integrative Plant Biology 48, 1473-1477.

Zhao Y, Chen P, Liao X, Zhou B, Liao J, Huang Z, Kong X, Zhou R. 2013. A comparative study of the atp 9 gene between a cytoplasmic male sterile line and its maintainer line and further development of a molecular marker specific for male sterile cytoplasm in kenaf (Hibiscus cannabinus L.). Molecular Breeding 32, 969-976.

Zhao Y, Liao X, Huang Z, Chen P, Zhou B, Liu D, Kong X, Zhou R. 2015. Expression of kenaf mitochondrial chimeric genes HM184 causes male sterility in transgenic tobacco plants. Mitochondrial DNA 26, 495-500.

Zhao Y, Liao X, Zhou B, Zhao H, Zhou Y, Zhou R. 2016. Mutation in the coding sequence of atp6 are associated with male sterile cytoplasm in kenaf (Hibiscus cannabinus L.). Euphytica 
207, 169-175.

Zhou RY. 2002. Discovery of Male Sterile Plants in Kenaf (Hibscus cannabinus L.). Scientia Agricultura Sinica 2, 212 (in Chinese).

Zhou RY, Zhang X, Zhang JQ, Gan ZH, Wei HX. 2008. A Breakthrough in kenaf cytoplasmic male sterile lines breeding. Scientia Agricultura Sinica 41, 314 (in Chinese). 